\title{
Integrating patient safety into the clinical microsystem
}

\author{
J J Mohr, P Batalden, P Barach
}

Qual Saf Health Care 2004;13(Suppl II):ii34-ii38. doi: 10.1136/qshc.2003.009571

Healthcare institutions continue to face challenges in providing safe patient care in increasingly complex organisational and regulatory environments while striving to maintain financial viability. The clinical microsystem provides a conceptual and practical framework for approaching organisational learning and delivery of care. Tensions exist between the conceptual theory and the daily practical applications of providing safe and effective care within healthcare systems. Healthcare organisations are often complex, disorganised, and opaque systems to their users and their patients. This disorganisation may lead to patient discomfort and harm as well as much waste. Healthcare organisations are in some sense conglomerates of smaller systems, not coherent monolithic organisations. The microsystem unit allows organisational leaders to embed quality and safety into a microsystem's developmental journey. Leaders can set the stage for making safety a priority for the organisation while allowing individual microsystems to create innovative strategies for improvement.

See end of article for authors' affiliations

\section{Correspondence to:} AssistantProfessor JJMohr, Department of Medicine, University of Chicago, 5841 S. Maryland Avenue, MC 2007 Chicago, IL 60637, USA. jmohr@medicine.bsd. uchicago.edu
A clinical microsystem is a group of clinicians and staff working together with a shared clinical purpose to provide care for a population of patients. ${ }^{1-3}$ The clinical purpose and its setting defines the essential components of the microsystem. These include the clinicians and support staff, information and technology, the specific care processes, and the behaviours that are required to provide care to its patients. Microsystems evolve over time, responding to the needs of their patients, providers, and external pressures. They coexist with other microsystems within a larger (macro) organisation. Examples of clinical microsystems include a cardiovascular surgical care team, a community based outpatient care centre, or a neonatal intensive care unit. Each example has in common core elements: a focused type of care, clinicians and staff with the skills and training needed to engage in the required care processes, a defined patient population, and a certain level of information and technology to support their work. The core elements are consistent across these examples. What often differs across microsystems is the ability of individual caregivers to recognise their efforts as part of a microsystem as well as the microsystem's level of functioning.

The purpose of this paper is to provide an overview of the clinical microsystem and the relationship between the macrosystem and microsystem, to address how the microsystem concepts can enhance the quality and safety of care on the frontlines, and to discuss how to facilitate learning and collaboration across microsystems.

\section{OVERVIEW OF CLINICAL MICROSYSTEMS}

The conceptual theory of the clinical microsystem is based on ideas developed by Deming, Senge, Wheatley, and others who applied systems thinking to organisational development, leadership, and improvement. ${ }^{4-6}$ The seminal idea for the clinical microsystem stems from the work of Quinn, based on analysing the world's best of best service organisations, such as FedEx, Mary Kay Cosmetics, McDonald's, and Nordstrom. ${ }^{7}$ He focused on determining what these extraordinary organisations were doing to achieve high quality, explosive growth, high margins, and excellent customer reputation. He found that these leading service organisations organised around, and continually engineered, the frontline relationships that connected the needs of customers to the organisation's core competency. Quinn called this frontline activity that embedded the service delivery process the smallest replicable unit or the minimum replicable unit. The smallest replicable unit, or the microsystem, is the key to implementing effective strategy, information technology, and other key aspects of intelligent enterprise.

In the late 1990s, Mohr and Donaldson investigated high performing clinical microsystems. ${ }^{2} 8$ Their research was based on a national search for the highest quality microsystems in health care. Forty three clinical units were identified using theoretical sampling and their leaders were interviewed using a semi-structured interview protocol. The results of the interviews were analysed to determine the characteristics that seemed most responsible for enabling these microsystems to be effective. The results suggested that eight dimensions were associated with high quality of care (see box 1). Further research on clinical microsystems provided more indepth case studies of 20 high performing microsystems and included on site interviews with every member of these microsystems and an analysis of the individual microsystem's performance data. ${ }^{9-17}$ A microsystem assessment tool, based on these eight dimensions, was developed to translate this research into practice, by giving clinicians and staff a way to gauge the level of functioning of their microsystem and then to help identify areas for improvement. ${ }^{18}$

Systems, in general, often bring up images of well oiled machines. In actuality, much of what we know and have experienced in health care does not match this description. Healthcare 
Box 1. Eight dimensions of high performing microsystems

- Constancy of purpose

- Investment in improvement

- Alignment of role and training for efficiency and staff satisfaction

- Interdependence of the care team to meet patient needs

- Integration of information and technology into work flows

- Ongoing measurement of outcomes

- Supportiveness of the larger organisation

- Connection to the community to enhance care delivery and extend influence

systems are cumbersome, unwieldy, unfriendly, and opaque to the users and the patients. Healthcare systems are better described as complex adaptive systems. As such, they are a collection of individual healthcare professionals who are free to act in ways that are not totally predictable; the organisational boundaries are fuzzy, in that membership changes and providers can simultaneously be members of other systems. Furthermore, given the complexity of these systems, the actions of individuals are interconnected so that the actions of one provider changes the context for all of the other providers. $^{19} 20$

As we continue to move beyond conceptual theory and research to clinical applications, the emerging fields of chaos theory, complexity science, and complex adaptive systems have influenced how we have applied these concepts to improving microsystems. ${ }^{19-22}$ This is evident in the work to bring together microsystems from around the world to learn and share best practices (see http://clinicalmicrosystem.org).

\section{Microsystem $v$ macrosystem}

Healthcare organisations are composed of multiple, differentiated, autonomous microsystems. Several assumptions are made about the relationship between the microsystem and the macrosystem?:

- Bigger systems (macrosystems) are made of smaller systems

- These smaller systems (microsystems) produce quality, safety, and cost outcomes at the frontline of care

- Ultimately the outcomes of the macrosystems can be no better than the microsystems on which they are formed

- The loyalty of most microsystem providers is first and foremost to their patients and to the microsystem and rarely to the larger macrosystem.

This requires interventions within all microsystems in the organisation if the organisation as a whole wants to improve. However, this doesn't mean that the microsystem functions independently from the other microsystems it regularly works with, or its macrosystem-indeed the microsystems within the macro-organisation are interconnected. The role of leadership is to set the general tone of the organisation, to facilitate the interconnections between the microsystems, and to cultivate learning disciplines in the organisation. Finally, senior leaders can pay careful attention to the questions they ask to hold the microsystems accountable for their performance to provide safer care (see box 2). They must realise the unintended consequences of mandating new rules and regulations but not respecting the ecology of the microsystem. ${ }^{23}$
Box 2. Questions senior leaders could ask about patient safety

- What information do we have about errors and patient harm?

- What is the patient safety plan?

- How will the plan be implemented at the organisational level and at the microsystem level?

- What type of infrastructure is needed to support implementation?

- What is the best way to communicate the plan to the individual microsystems?

- How can we foster reporting (telling the truth) about patient harm?

- How will we empower microsystem staff to make suggestions for improving safety?

- How can we build linkages to the key stakeholders?

- What stories can we tell that relate the importance of patient safety?

- How will we recognise and celebrate progress?

- What are the unintended consequences of new actions, rules, and guidelines?

\section{Improving safety within the clinical microsystem}

Patient safety has been defined as freedom from accidental injury. ${ }^{24}$ Accidental injury can be due to error that includes the failure of a planned action to be completed as intended or the use of a wrong plan to achieve an aim. ${ }^{24}{ }^{25}$ Various organisations have accepted the call to action to make patient safety a priority. For example, in the United States the Joint Commission on the Accreditation of Healthcare Organizations (JCAHO) has developed programme specific patient safety goals. ${ }^{26}$ As we go indepth in one specific area, the recently released 2005 JCAHO goals for hospitals include improving the accuracy of patient identification, the effectiveness of communication among caregivers, the safety of using medications and infusion pumps, and reducing the risk of healthcare associated infections. ${ }^{26}$ How can an organisation respond to the need to meet patient safety goals?

We believe that the first step is the realisation that safety is a property of the clinical microsystem that can be achieved only through a systematic application of a broad array of process, equipment, organisation, supervision, training, and teamwork changes. The clinical microsystem-as a unit of research, analysis, and practice-is an important level at which to focus patient safety interventions. It is at this systems level that most patients and caregivers meet, and it is at this level that real changes in the patient care usually given can (and must) be made. Errors occur within the microsystem and ultimately it is the functioning microsystem that can stop, prevent, or mitigate errors from causing patient harm. Many methods are available to explore the causal system at work ${ }^{27-31}$ including probability analysis ${ }^{29}$ and near miss analysis. ${ }^{32}$ These all suggest the importance of analysing the entire causal system, not just seeking a root cause.

Table 1 builds on the research of high performing microsystems and provides specific actions that can be further explored. This list provides an organising framework to apply safety concepts to the microsystem.

\section{PROMOTING SYSTEM RESILIENCE ACROSS AND BETWEEN THE MICROSYSTEMS}

Microsystems usually coexist with multiple other microsystems within the organisation. Patients are aware of the gaps 


\begin{tabular}{|c|c|}
\hline Microsystem characteristics & What this means for patient safety \\
\hline Leadership & $\begin{array}{l}\text { Define the safety vision of the organisation } \\
\text { Identify the existing constraints within the organisation } \\
\text { Allocate resources for plan development, implementation, and ongoing } \\
\text { monitoring and evaluation } \\
\text { Build in microsystems participation and input to plan development } \\
\text { Align organisational quality and safety goals } \\
\text { Engage the Board of Trustees in ongoing conversations about the } \\
\text { organisational progress toward achieving safety goals } \\
\text { Recognition for prompt truth telling about errors or hazards } \\
\text { Certification of helpful changes to improve safety }\end{array}$ \\
\hline Organisational support & $\begin{array}{l}\text { Work with clinical microsystems to identify patient safety issues and make } \\
\text { relevant local changes } \\
\text { Put the necessary resources and tools into the hands of individuals }\end{array}$ \\
\hline Staff focus & $\begin{array}{l}\text { Assess current safety culture } \\
\text { Identify the gap between current culture and safety vision } \\
\text { Plan cultural interventions } \\
\text { Conduct periodic assessments of culture } \\
\text { Celebrate examples of desired behaviour - for example, acknowledgement of } \\
\text { an error }\end{array}$ \\
\hline Education and training & $\begin{array}{l}\text { Develop patient safety curriculum } \\
\text { Provide training and education of key clinical and management leadership } \\
\text { Develop a core of people with patient safety skills who can work across } \\
\text { microsystems as a resource }\end{array}$ \\
\hline Interdependence of the care team & $\begin{array}{l}\text { Build plan-do-study-act (PDSA) into debriefings } \\
\text { Use daily huddles to debrief and to celebrate identifying errors }\end{array}$ \\
\hline Patient focus & $\begin{array}{l}\text { Establish patient and family partnerships } \\
\text { Support disclosure and truth around medical error }\end{array}$ \\
\hline Community and market focus & $\begin{array}{l}\text { Analyse safety issues in community and partner with external groups to reduce } \\
\text { risk to population }\end{array}$ \\
\hline Performance results & $\begin{array}{l}\text { Develop key safety measures } \\
\text { Create feedback mechanisms to share results with microsystems }\end{array}$ \\
\hline Process improvement & $\begin{array}{l}\text { Identify patient safety priorities based on assessment of key safety measures } \\
\text { Address the work that will be required at the microsystem level }\end{array}$ \\
\hline $\begin{array}{l}\text { Information and information } \\
\text { technology }\end{array}$ & $\begin{array}{l}\text { Enhance error reporting systems } \\
\text { Build safety concepts into information flow (for example, checklists and } \\
\text { reminder systems) }\end{array}$ \\
\hline
\end{tabular}

and hand offs between microsystems as they navigate the healthcare system, for example as they transfer from inpatient care back into the community. These crossmicrosystem relationships will be essential to improving hand offs but also to providing opportunities for learning about systemic problems within the institution and interventions to improve quality and safety. We might learn something useful about how to facilitate the interaction of different microsystems by examining the factors that lead to success in other types of collaborative relationships, such as community health partnerships.

An effective collaborative relationship is based on the underlying assumption that collaboration is a more effective approach to achieve a goal than multiple individual efforts. Weick suggests that leaders today need to develop groups that are respectful of the interactions that hold the group together. ${ }^{33}$ Resilient groups have respectful interactions that are founded on three major elements ${ }^{35}$ :

- Trust-a willingness to base beliefs and actions on the reports of others

- Honesty-reporting so that others may use your observations in developing and enhancing their own beliefs

- Self-respect-integrating one's perceptions and beliefs with the reports of others without depreciating them or yourself.

Kaluzny $^{36}$ discusses the conditions that must be present for relationships across organisations to develop. For voluntary interactions-which may be quite different than those mandated by an external power-several conditions must be met. There must be an internal need for resources, a commitment to an external problem, and the opportunity to change. In addition, there must be a consensus on the external problem(s) facing the organisations as well as a consensus on the specific goals and services for developing a joint effort. The 1999 Institute of Medicine report To err is human, for example, brought patient safety to the forefront of the agenda as well as set the stage for discussing specific goals and strategies for achieving those goals. ${ }^{37}$

Mitchell and Shortell provide a synthesis of the literature on success of community health partnerships to suggest factors that influence the success of interorganisational relations (box 3).

The Center for the Advancement of Collaborative Strategies in Health (CACSH) at The New York Academy of Medicine focused on strengthening the ability of partnerships to assess and achieve the unique advantage of collaboration-what they term partnership synergy. CACSH conducted a study to create and operationalise a framework for assessing partnership synergy and for identifying its likely determinants (box 4). ${ }^{38}$

According to CACSH, effective leadership facilitates productive interactions among partners by bridging diverse cultures, performing boundary spanning functions, and revealing as well as challenging assumptions that limit thinking and action.

Partnership efficiency refers to making good use of partners' time, in-kind resources, and financial resources. The effectiveness of a partnership's administration and management also has an impact on partnership synergy, although this effect was not statistically significant. Administration and management activities, such as coordination of communication among partners and partnership activities, and the preparation of materials that inform partners, are the glue that makes it possible for multiple 
Box 3. The successful interorganisational relationship

- Context-refers to the environment in which the partnership exists-the internal and external stakeholders, their historical relationships and influence, the presence or absence of human and financial resources, the political environment, public sentiments, and the current challenges facing the community.

- Strategic intent-a similar concept to a consensus on the external problem(s) facing the organisations, refers to the reasons the interorganisational relationship is formed.

- Resource base-a diversified resource base helps assure that the collaborative is able to pursue their strategic intent without getting sidetracked by pursuing the goals of a single funding agency.

- Membership heterogeneity-refers to the balance of the participating members in regard to the number and types of participants.

- Coordination skills-informal as well as formal communication mechanisms assure that the collaborators meet their own goals and are held accountable to demonstrate their progress internally and externally.

- Response to accountability.

\section{Box 4. Six dimensions of partnership synergy}

- Effectiveness of leadership

- Effectiveness of administration and management

- Sufficiency of intangible resources

- Partnership efficiency

- Challenges related to partner involvement

- Challenges related to the community

independent people and organisations to work together. Analysis results indicate that the sufficiency of non-financial resources influences, although non-significantly, partnership synergy. Non-financial resources such as skills and expertise, information, and connectivity to target populations play a unique role; synergy is largely built from these resources, and it is only by combining them in novel ways that partners can

\section{Implications for clinical practice}

- Characteristics of high performing microsystems provide a framework for applying specific interventions to improve the microsystem's ability to prevent or mitigate errors from causing patient harm.

- Engaging the Board of Trustees in ongoing conversations about the organisation's progress toward achieving quality and safety goals is important to the success of the organisation.

- Increasing opportunities for cross-microsystem learning is essential for learning about the systemic problems within the institution and interventions to improve quality and safety.

\section{Key messages}

- The clinical microsystem, as a unit of research, analysis, and practice, is an important level at which to focus patient safety interventions.

- A functioning microsystem can stop, prevent, or mitigate errors from causing patient harm.

- Safety is a property of the clinical microsystem that can only be achieved through a systematic application of a broad array of process, equipment, organisation, supervision, training, simulation, and teamwork changes.

potentially create something that enables them to accomplish more than they could on their own.

\section{CONCLUSIONS}

The microsystem concepts have evolved from systems theory and primary research on characteristics of high performing clinical units. Specific interventions can be made to embed quality and safety into the microsystem. Although we offer several suggestions related to each of the microsystem characteristics, this list should only serve as a guiding framework to adapt to individual microsystems. Leaders should set the stage for making safety a priority for the organisation, but they should allow individual microsystems to create innovative strategies for improvement. Successful examples of improving safety and quality in microsystems will help to harvest the experiences in cross microsystem colloboratives. Healthcare organisations are not monolithic, but composed of smaller microsystems, so interventions need to be expressed at the microsystem level. This requires attention to detail in redesign of health care. Fostering collaborative relationships among microsystems should be an important goal of organisations. Microsystems can help to organise and design resilience into the communications, work processes, and clinical environments of complex healthcare organisations.

\section{Authors' affiliations}

J Mohr, Department of Medicine, University of Chicago, Chicago, USA P Batalden, Department of Pediatrics, Community and Family Medicine, Dartmouth Medical School, Dartmouth, USA

P Barach, Department of Anesthesiology, Miami Center for Patient Safety, University of Miami, Miami, USA

\section{REFERENCES}

1 Batalden PB, Mohr JJ, Nelson EC, et al. Continually improving the health and value of health care for a population of patients: the panel management process. Qual Manag Health Care 1997;5:41-51.

2 Mohr J. Forming, operating, and improving microsystems of care. Hanover: Center for the Evaluative Clinical Sciences, Dartmouth College, 2000:250.

3 Nelson EC, Batalden PB, Mohr JJ, et al. Building a quality future. Front Health Serv Manage 1998;15:3-32.

4 Deming WE. Out of the crisis. Cambridge: Massachusetts Institute of Technology Center for Advanced Engineering Study, 1986.

5 Senge P. The fifth discipline. New York, NY: Doubleday, 1990.

6 Wheatley M. Leadership and the new science: learning about organization from an orderly universe. San Francisco, CA: Berrett-Koehler, 1992.

7 Quinn JB. The intelligent enterprise. New York, NY: Free Press, 1992.

8 Donaldson MS, Mohr JJ. Improvement and innovation in health care microsystems. A technical report for the institute of medicine committee on the quality of health care in America. Princeton, NJ: Robert Wood Johnson Foundation, 2000

9 Nelson E, Batalden PB, Huber TP, et al. Microsystems in health care: part 1. Learning from high-performing front-line clinical units. Jt Comm J Qual Saf 2002;28:472-93.

10 Nelson EC, Batalden PB, Homa K, et al. Microsystems in health care: part 2. Creating a rich information environment. Jt Comm J Qual Saf 2003;29:5-15. 
11 Godfrey MM, Nelson EC, Wasson JH, et al. Microsystems in health care: part 3. Planning patient-centered services. Jt Comm J Qual Saf 2003;29:159-70.

12 Wasson JH, Godfrey MM, Nelson EC, et al. Microsystems in health care: part 4. Planning patient-centered care. Jt Comm J Qual Saf 2003:29:227-37.

13 Batalden PB, Nelson EC, Mohr JJ, et al. Microsystems in health care: part 5. How leaders are leading. Jt Comm J Qual Saf 2003;29:297-308.

14 Mohr J, Barach P, Cravero JP, et al. Microsystems in health care: part 6. Designing patient safety into the microsystem. It Comm J Qual Saf 2003;29:401-8.

15 Kosnik L, Espinosa J. Microsystems in health care: part 7. The microsystem as a platform for merging strategic planning and operations. Jt Comm J Qual Saf 2003;29:452-9.

16 Huber T, Godfrey MM, Nelson EC, et al. Microsystems in health care: part 8 . Developing people and improving worklife: what front-line staff told us, Jt Comm J Qual Saf 2003;29:512-22.

17 Batalden P, Nelson EC, Edwards WH, et al. Microsystems in health care: part 9. Developing small clinical units to attain peak performance. Jt Comm J Qual Saf 2003;29:575-85.

18 Mohr J, Batalden P. Improving safety at the front lines: the role clinical microsystems. Qual Saf Health Care 2002;11:45-50.

19 Plsek PE, Greenhalgh T. Complexity science: the challenge of complexity in health care. BMJ 2001;323:625-8.

20 Plsek PE, Wilson T. Complexity, leadership, and management in healthcare organisations. BMJ 2001;323:746-9.

21 Arrow H, McGrath J, Berdahl J. Small groups as complex systems. Thousand Oaks: Sage Publications, 2000.

22 Peters T. Thriving on chaos: handbook for a management revolution. New York: Harper \& Row, 1987.

23 Amalberti R. The paradoxes of almost totally safe transportation systems. Saf Sci 2001;37:109-26.

24 Institute of Medicine. To err is human-building a safer health system. Washington: National Academy Press, 1999.

25 Reason J. Human error. New York, NY: Cambridge University Press, 1990.
26 Joint Commission on Accreditation of Healthcare Organizations. National patient safety goals for 2004 and 2005. Oakbrook Terrace, IL: JCAHO, 2004. www.jcaho.org/accredited+organizations/patient+safety/npsg.htm (accessed 24 July 2004).

27 Vincent C. Understanding and responding to adverse events. N Engl J Med 2003;348: 1051-6.

28 Vincent C, Taylor-Adams S, Stanhope N. Framework for analysing risk and safety in clinical medicine. BMJ 1998:316:1154-7.

29 Apostalskis G, Barach P. Lessons learned from nuclear power. In: Hatlie M, Tavill K, eds. Patient safety: international textbook. Faithersburg, MD: Aspen Publications, 2004:205-225.

30 Dekker S. The field guide to human error investigations. Aldershot: Ashgate Publishing Limited, 2002.

31 Reason J. Understanding adverse events: human factors. In: Vincent C, ed. Clinical risk management. London: BMJ Publications, 1995:31-54.

32 Van der Schaff T. Development of a near miss management system at a chemical process plant. In: Lucas D, ed. Near miss reporting as a safety tool. Butterworth-Heinemann: Oxford, 1991.

33 Weick KE. The collapse of sensemaking in organizations: the Mann Gulch disaster. Adm Sci Q 1993:38:628-52.

34 Weick KE. Sensemaking in organizations. Thousand Oaks, CA: Sage Publications, 1995.

35 Weick KE. Prepare your organization to fight fires. Harvard Business Review 1996:74:3-6.

36 Kaluzny A. Design and management of disciplinary and interdisciplinary groups in health services: review and critique. Med Care Rev 1985;42:77-1 12

37 Kohn L, Corrigan J, Donaldson M, eds. To err is human-building a safer health system. Washington: National Academy Press, 1999.

38 Lasker RD, Weiss ES, Miller R. Partnership synergy: a practical framework for studying and strengthening the collaborative advantage. Milbank $Q$ 2001;79:179-205. 